Résumés des conférences et travaux

\title{
Droits du Proche-Orient ancien
}

Conférences de l'année 2014-2015

\section{Sophie Démare-Lafont}

\section{(2) OpenEdition \\ Journals}

\section{Édition électronique}

URL : https://journals.openedition.org/ashp/1803

DOI : 10.4000/ashp.1803

ISSN : 1969-6310

\section{Éditeur}

Publications de l'École Pratique des Hautes Études

Édition imprimée

Date de publication : 1 septembre 2016

Pagination : 13-16

ISSN : 0766-0677

Référence électronique

Sophie Démare-Lafont, " Droits du Proche-Orient ancien », Annuaire de l'École pratique des hautes études (EPHE), Section des sciences historiques et philologiques [En ligne], 147 | 2016, mis en ligne le 21 septembre 2016, consulté le 06 juillet 2021. URL : http://journals.openedition.org/ashp/1803 ; DOI : https://doi.org/10.4000/ashp.1803 


\title{
DROITS DU PROCHE-ORIENT ANCIEN
}

\author{
Directeur d'études : M ${ }^{\text {me }}$ Sophie DÉmare-Lafont
}

Programme de l'année 2014-2015 : Les grands textes législatifs mésopotamiens.

La conférence de cette année a entamé un cycle consacré à l'étude des grands recueils législatifs du Proche-Orient ancien. C'est le Code de Hammurabi qui a été choisi pour ouvrir ce cycle. Il constitue en effet le monument le plus célèbre mais aussi le plus abouti du genre, et nous a été transmis dans sa forme officielle et quasi-complète.

Après une introduction générale portant sur les sources législatives cunéiformes et la discussion autour de leur valeur juridique, on a commencé la lecture du Code paragraphe par paragraphe, en laissant de côté le prologue, partie plus littéraire et moins technique, dont la valeur dispositive est cependant très probable.

Dans la mesure du possible, on a essayé de rapprocher les normes législatives des actes de la pratique contemporains ou de sources postérieures, moins pour mesurer l'effectivité du Code dans la vie juridique (le débat a été abordé dans l'introduction) que pour éclairer certaines questions soulevées par la lecture des cas présentés et des solutions choisies.

Le $\S 1^{\text {er }}$, portant sur l'accusation infondée de meurtre, soulève une interrogation à propos de l'administration de la preuve. Le verbe kunnum (1. 30 la uktīnšu « il n'a pas rapporté la preuve contre lui ») se rapporte à la preuve testimoniale (cf. CAD kânu A 4a p. 168) : celui qui intente l'action pour meurtre ne trouve donc pas de témoins pour soutenir ses allégations et il subit alors la peine encourue par l'accusé, à savoir la mort. Mais on peut se demander pourquoi, compte tenu de la gravité de l'affaire, le juge n'a pas eu recours à une preuve surnaturelle. Dans une situation similaire, plusieurs siècles après, un texte apologétique raconte comment le roi néo-babylonien Nabuchodonosor soumit à l'ordalie du fleuve les deux protagonistes d'un procès en homicide. Mais la procédure prit un tour inattendu puisque l'accusateur coula et se noya, son corps demeurant même introuvable quelques temps. Il est difficile de comprendre pourquoi, dans le cas visé par Hammurabi, le serment ou l'ordalie fluviale ne sont pas envisagés, et ce d'autant plus que le $\S 2$ du Code, visant la fausse accusation de sorcellerie, prévoit justement le recours au dieu-Fleuve face à l'absence de preuve matérielle. Mais le risque de représailles, tenu pour un juste motif d'abstention dans ce cas, ne pèse pas sur les témoins d'un meurtre ; dès lors, l'homme qui ne peut faire corroborer son accusation par des tiers est présumé agir par malveillance.

$\mathrm{Au} \S 2$, l'individu accusé de sorcellerie qui n'est pas confondu par des témoins (1. 36 la uktīnšu) doit s'immerger dans le dieu-Fleuve; s'il coule, prouvant ainsi sa culpabilité, son adversaire récupèrera tous ses biens ; s'il est blanchi par l'épreuve, il prendra les biens de l'accusateur, qui sera exécuté. La mise à mort du sorcier n'est pas expressément prévue par le texte, mais elle est induite par le parallélisme avec la sanction frappant le faux accusateur. Il faut sans doute comprendre que le patient a coulé au fond du fleuve et s'est noyé, autrement dit que l'ordalie a tourné à l'exécution 
du fautif, comme une sorte de châtiment immanent. L'absence du verbe ubbir « il a accusé ", laisse supposer que l'imputation n'a pas été faite devant un tribunal mais peut-être lancée au cours d'une dispute ou à l'occasion d'un autre procès (en ce sens, R. Westbrook, "Witchcraft and the Law in the Ancient Near East », dans J. Hengstl et U. Sick [éd.], Recht gestern und heute. Festschrift zum 85. Geburtstag von Richard Haase, Wiesbaden, 2006, p. 45-52). Cette seconde option est effectivement documentée dans deux lettres paléo-babyloniennes (AbB 9268 et 269) évoquant un litige entre un fils et son père, lequel parle avec mépris de l'épouse et de la belle-mère de son fils qu'il appelle « tes sorcières » (AbB 9268 : 24 ; cf AbB 9269 : 14).

Les $\S 3-4$ abordent le thème du faux témoignage. Comme pour la fausse accusation, la déposition mensongère ou calomnieuse est passible de la peine encourue par l'accusé. Cette similitude pose la question du statut du témoin, qui apparaît à beaucoup d'égard comme un accusateur. Pour illustrer cette ambiguïté, on a étudié une condamnation prononcée par les juges de Sippar (CBS 349 ; édition et bibliographie sur le site Archibab) contre deux religieuses convaincues de faux témoignage : elles sont frappées au visage jusqu'au sang et leur voile est arraché.

Le $\S 5$ concerne la forfaiture du juge qui, après avoir tranché un litige et établi une tablette scellée, a changé sa décision. On comprend habituellement que la loi punit une altération du document contenant le jugement, mais cette interprétation suppose que la falsification ait été opérée juste après la rédaction du texte et avant qu'il ne soit remis au vainqueur du procès, qui le conserve dans ses archives. Une autre hypothèse, suggérée par E. Szlechter (Codex Hammurapi, Rome, 1977, p. 32-33) serait que le juge, saisi une seconde fois des mêmes faits, a rendu une sentence inverse à la précédente, au mépris du principe d'autorité de la chose jugée. La concussion est un thème évoqué dans plusieurs lettres paléo-babyloniennes, notamment AbB 2 11, écrite par Hammurabi à Šamaš-hazir, à propos de la corruption du personnel administratif de Bad-tibira.

La séquence suivante du Code (§ 6-14) est consacrée au vol. Les $\S 6$ et 8 portent sur le vol de biens appartenant au temple ou au palais. Le $\S 6$ punit de mort le voleur et le receleur tandis que le $\S 8$ impose une amende au décuple ou au trentuple, selon que le bien volé appartient à un particulier ou à une institution; le voleur insolvable est tué. L'agencement de ces deux paragraphes, apparemment complémentaires mais séparés par le $\S 7$ consacré au dépôt (voir infra), pose problème. De même, on observe que les biens volés au $\S 6$ sont indifférenciés (NÍG.GA = makkūru 1. 32) alors qu'ils sont précisément énumérés au $\S 8$ (bœuf, mouton, âne, cochon, bateau). On a expliqué ces incohérences par une évolution du droit (San Nicolò, Westbrook), la version ancienne du $\S 6$ ayant été réformée au $\S 8$, ou par une distinction entre biens sacrés et biens profanes (Driver et Miles, Petschow, Koschaker) déterminée par le lieu où ils se trouvaient : à l'intérieur du temple ou du palais $(\S 6)$, le voleur connaissait forcément le statut particulier des objets qu'il a pris ; ailleurs, il pouvait l'ignorer ( 8 8). Selon une hypothèse suggérée par B. Fiette, la sévérité accrue du $\S 6$ s'expliquerait par la condition du voleur, membre du personnel du temple ou du palais, par opposition au coupable extérieur à ces institutions au $\S 8$. Un cas de vol de bétail du temple par un berger et deux complices extérieur (AbB 1341 ) a été étudié pour illustrer ce dossier.

$\mathrm{Au} \S 7$, le Code punit de mort l'individu qui a acheté ou pris en dépôt des choses fongibles des mains d'un fils de famille ou d'un esclave. L'insertion de ce texte dans 
la série sur le vol est appelée par l'occurrence du terme «voleur» (šarraqum 1. 56) pour qualifier le fautif. L'expression balum š̄ibi u riksātim a été discutée pour savoir si le $u$ est conjonctif ( «ni ») ou disjonctif ( ou »), avec les conséquences que cela entraîne pour le régime de la preuve du contrat. La loi impose la présence de témoins et la rédaction d'une tablette pour la vente ou le dépôt irrégulier effectués par le fils de famille ou l'esclave, afin de garantir qu'ils agissent pour le père ou le maître dans le cadre d'un mandat ou d'une gestion d'affaire. L'acquéreur qui ne respecte pas ces formes est réputé de mauvaise foi et mis à mort. Les dispositions du Code sur le même thème (§ 122-125) et celles des Lois d'Eshnunna ( $§ 15-16$ et 36-37) ont été examinées. B. Fiette a versé au débat un intéressant contrat des archives de Šamaš-hazir (OECT 8 13) qui est défini comme une location sur la tablette intérieure et un dépôt sur l'enveloppe.

Les $\S 9-13$ portent sur les modalités de la preuve de la propriété d'un bien meuble, perdu par son propriétaire et vendu par celui qui a trouvé l'objet. L'acquéreur prouve sa bonne foi en citant son auteur et ses témoins, auquel cas le vendeur est considéré comme voleur; il est tué et le bien est rendu à son propriétaire (§9). Si le détenteur $(\S 10)$ ou le revendiquant $(\S 11)$ ne peuvent prouver leurs droits, ils sont déclarés voleurs et tués. La mort du vendeur n'éteint pas l'obligation de remboursement, qui grève le patrimoine successoral ( $§ 12)$. L'éloignement des témoins du revendiquant (ou de l'acquéreur?) donne lieu à une prorogation de 6 mois pour leur comparution; passé ce délai, sa mauvaise foi est établie et le rend passible d'une peine (§ 13). Quelques affaires de vol d'animaux à Ur III (MVN 3 219) et à l'époque paléo-babylonienne (YOS 8159 ; Sumer $14 n^{\circ} 28$ ) ont été choisis pour éclairer ces paragraphes.

Le $\S 14$ clôture cette série sur le vol en punissant le rapt d'un enfant par la peine de mort. Ces enlèvements sont attestés dans plusieurs documents paléo-babyloniens. À titre d'exemple, on a lu la lettre AbB 1321.

Ce texte fait une transition avec le thème de la série suivante $(\S 15-20)$ consacrée au kidnapping ou à la fuite des esclaves. Le phénomène est très fréquent en Mésopotamie, et notamment à l'époque paléo-babylonienne, comme le montrent les clauses des contrats de vente servile, mais aussi de garde ou de dépôt, à l'instar de VS 8 123124. Le Code punit de mort celui qui fait sortir de la ville un ou une esclave qui ne lui appartient pas ( $§ 15$ ) ou héberge un fugitif ( $\$ 16$ et 19); une prime est prévue pour celui qui ramène à son maître un fuyard $(\S 17)$; c'est au palais qu'il incombe d'enquêter en cas de difficulté pour identifier le propriétaire $(\S 18)$; enfin, le détenteur auquel l'esclave a échappé avant d'être remis à son maître, jure sa bonne foi pour écarter la suspicion de recel $(\$ 20)$. Plusieurs de ces lois avaient déjà été étudiées en 2013-2014, en parallèle avec les $\S 51-52$ des Lois d'Eshnunna. On a enrichi le commentaire avec la lecture de la lettre AbB 7 116, concernant le vol dans une place forte de divers biens de valeur, dont des esclaves.

Les $§ 21-25$ incriminent divers types de vol aggravé : cambriolage avec effraction (§ 21), vol avec violence ( $(22)$, vol dans une maison en feu ( $\$ 25)$. L'indemnisation est à la charge de la collectivité si le voleur n'est pas attrapé, que le dommage porte sur des biens ( $(23)$ ou des vies ( $(24)$.

On a terminé l'année par la lecture des $\S 26-41$, qui détaillent le régime juridique des tenures attribuées aux soldats. Le commentaire, déjà élaboré dans une étude de 
synthèse sur la notion de fief (cf. S. Démare-Lafont, « Fief et féodalité dans le ProcheOrient ancien », dans J.-P. Poly et E. Bournazel [éd.], Les féodalités, Paris, 1998, p. 515-630, spécial. p. 541-546), a été complété par la lecture de deux actes de la pratique : un document de partage successoral (CT 8 3a), et un décompte des jours de service dus par un tenancier (MAH 16281). 